\title{
Shu Yu Wan Formula
}

National Cancer Institute

\section{Source}

National Cancer Institute. Shu Yu Wan Formula. NCI Thesaurus. Code C120207.

A traditional Chinese medicine comprising different herbs that may be used for a variety of medical purposes. Shu Yu Wan contains the following herbs: Da Zao (Fructus Jujubae), Shu Yu (Radix Dioscoreae Quinquelobae), Gan Cao (Radix Glycyrrhizae Uralensis), Shu Di Huang (Radix Rehmanniae Glutinosae Praeparata), Dang Gui (Radix Angelicae Sinensis), Shen Qu (Massa Medica Fermentata), Gui Zhi (Ramulus Cinnamomi Cassiae), Da Dou Juan (Semen Glycines Germinatum), E Jiao (Gelatinum Corii Asini), Ren Shen (Radix Ginseng), Bai Zhu (Rhizoma Atractylodis Macrocephalae), Fu Ling (Sclerotium Poriae Cocos), Chuan Xiong (Radix Ligustici Wallichii), Bai Shao Yao (Radix Paeoniae Lactiflorae), Mai Men Dong (T uber Ophiopogonis Japonici), Chai Hu (Radix Bupleuri), Fang Feng (Radix Ledebouriellae Divaricatae), Jie Geng (Radix Platycodi Grandiflori), Xing Ren (Semen Pruni Armeniacae), Bai Lian (Radix Ampelopsis Japonicae) and Sheng Jiang (Rhizoma Zing iberis Officinalis Recens). This formula may be used to relieve chemotherapeutic side effects or cancerrelated symptoms. 